Brit. Heart f., 1969, 31, 146.

\title{
Aortography in Fallot's Tetralogy and Variants
}

\author{
SIMON REES AND JANE SOMERVILLE \\ From The Institute of Cardiology and National Heart Hospital, London W.1
}

In patients with Fallot's tetralogy and variants, right ventricular angiocardiography is routinely used to demonstrate the basic anatomy. This investigation may fail to demonstrate clearly the aortic branches and the anatomy of the systemic arteries supplying the lungs. The contribution of aortography in the pre-operative investigation of these patients has been examined in detail.

\section{SUBJECTS AND METHODS}

Forty-one aortograms obtained in $\mathbf{4 0}$ patients were available for study. One patient had an aortogram before and after a shunt operation. The diagnosis was Fallot's tetralogy in 28, pulmonary atresia in 10, corrected transposition with pulmonary stenosis and ventricular septal defect in 1, and transposition with pulmonary atresia in 1 . In 36 investigations the catheter was passed from the saphenous vein through the right ventricle into the aorta, and in 5 retrograde arterial catheterization was used. 0.75 to $1.5 \mathrm{ml} . / \mathrm{kg}$. body weight of Conray 420 was injected under pressure into the ascending aorta at a rate of approximately $33 \mathrm{ml}$. per second. The dose of contrast medium was higher in patients with pulmonary atresia and in those who were thought to have a large aortopulmonary communication. Full-size films were exposed in antero-posterior and lateral projections at 6 or 12 frames per second. After the aortogram, the catheter tip was withdrawn to the right ventricle and a right ventricular angiocardiogram obtained in the usual way. The following structures were studied on the aortogram: surgical anastomoses, systemic to pulmonary collateral arteries, persistent ductus, the anatomy of the aortic root, the aortic branches including the coronary arteries, and the pulmonary arteries in pulmonary atresia. In addition, the presence or absence of an audible continuous murmur was correlated with the findings on aortography in an attempt to determine the clinical indications for this investigation.

\section{RESULTS}

Aortography added no morbidity to the investigation. One patient who developed a hemiplegia

Received September 3, 1968. three days after the procedure was found to have bacterial endocarditis which probably was present at the time of cardiac catheterization. In all patients the anatomical details of the aorta and its branches were clearer on the aortogram than when the contrast medium was injected into the right ventricle (Fig. 1, 2, and 3).

Surgical Anastomoses. Surgical anastomoses had been performed in 15 patients (left Blalock 5, right Blalock 2, bilateral Blalock 2, Waterston 3, Potts 3). Two patients had had two operations, making a total of 17 anastomoses, and of these 14 were shown on aortography to be patent and 3 were blocked (Fig. 4). It was difficult to be certain of patency from the right ventricular angiocardiogram alone in 7 patients. In the majority with a single patent anastomosis of any type, there was selective perfusion of the ipsilateral lung (Fig. 5). Only 3 patients had bilateral equal perfusion from one anastomosis (Fig. 6).

Apart from giving information about the functional state of a shunt, the aortogram was occasionally found to reveal unexpected anatomical findings. One such patient with a right aortic arch was said to have had a left subclavian-pulmonary anastomosis, but the aortogram showed that the innominate and not the subclavian artery had been anastomosed to the left pulmonary artery. Aortic filling on the right ventricular angiocardiogram was insufficient to show this (Fig. 3).

Systemic-pulmonary Collateral Arteries. Abnormal systemic-pulmonary collateral arteries were seen in 28 patients. Some enlargement of bronchial arteries, which were otherwise anatomically normal, was seen in 14 of the 17 patients with Fallot's tetralogy in whom there was neither a shunt nor other anomaly. In 5 patients with Fallot's tetralogy complicated by a blocked surgical anastomosis or a stenosed or occluded main pulmonary artery, the 146 


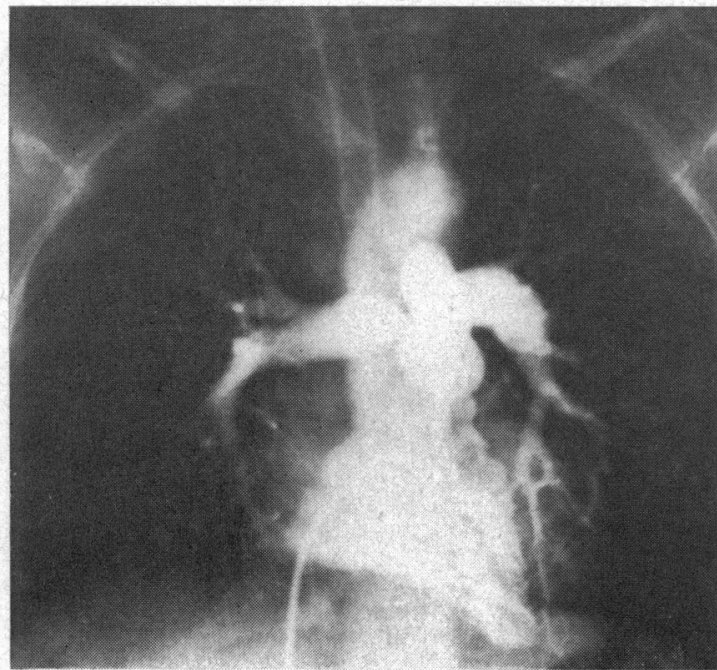

(a)

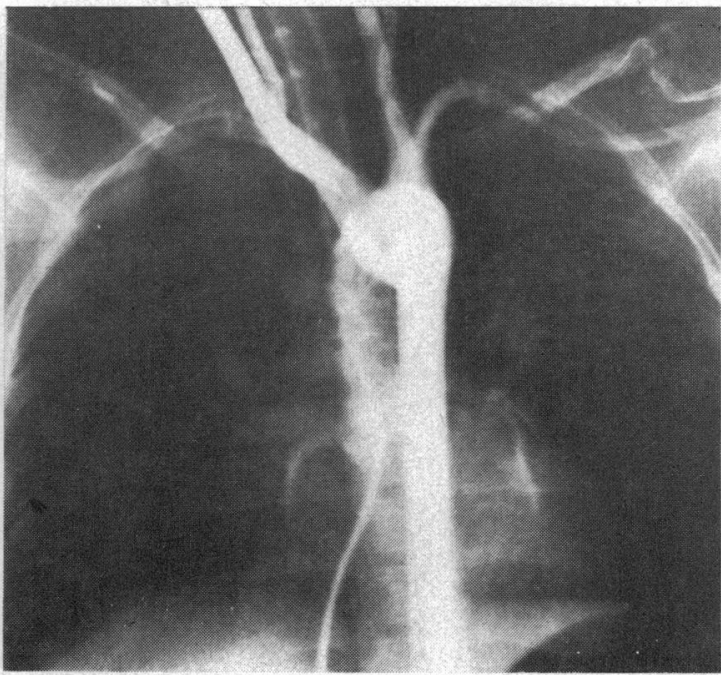

(b)

Fig. 1.-(a) Right ventricular angiocardiogram (frontal view) in patient with Fallot's tetralogy, showing poor aortic filling with aortic arch branches not visible. (b) Aortogram from the same patient showing aberrant right subclavian artery arising from the descending aorta.

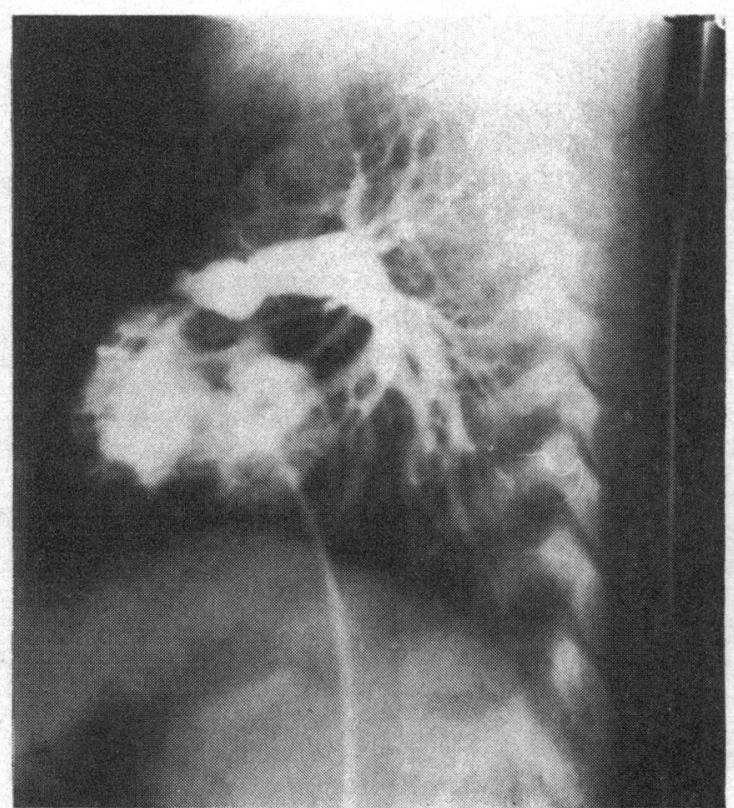

(a)

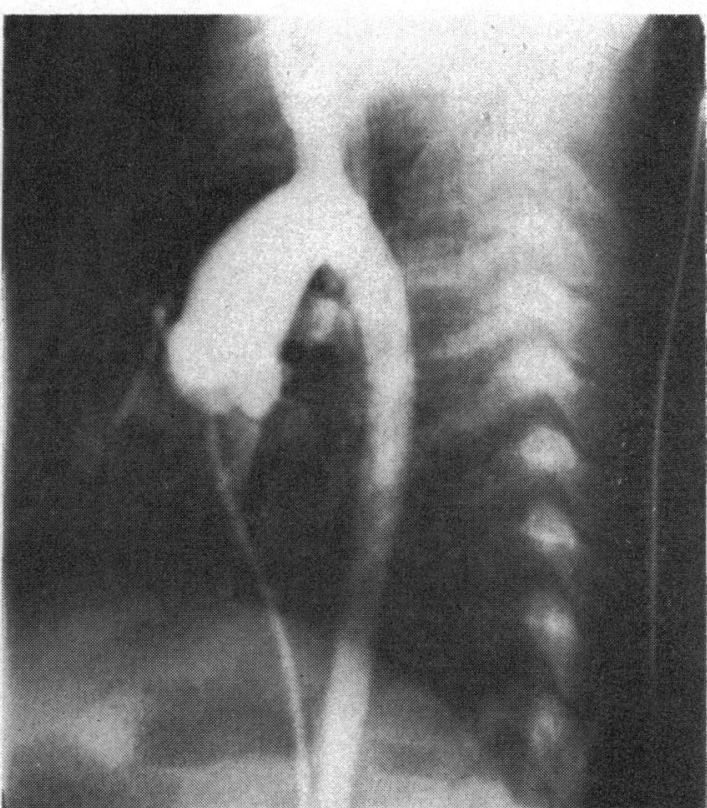

(b)

Fig. 2.-(a) Right ventricular angiocardiogram (lateral view) in patient with Fallot's tetralogy, showing poor aortic filling. (b) Aortogram (lateral view) showing small persistent ductus. No continuous murmur was audible. 


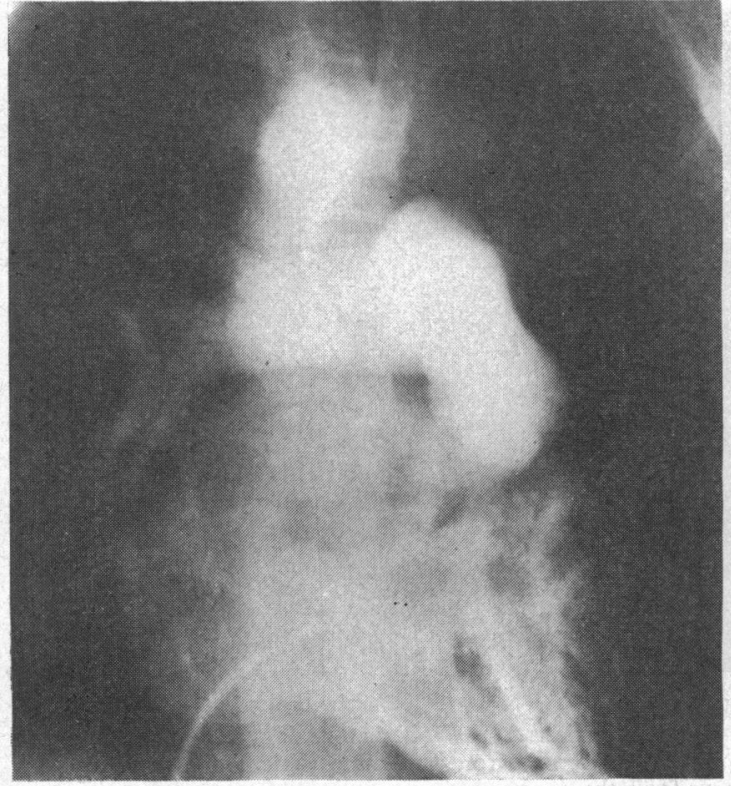

(a)

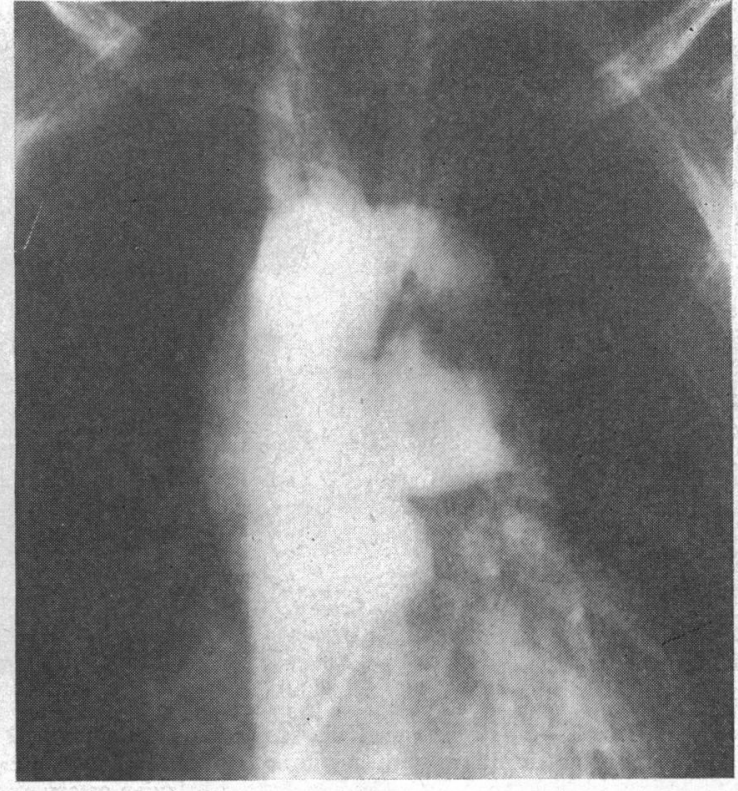

(b)

FIG. 3.-(a) Right ventricular angiocardicgram (frontal view) in patient with Fallot's tetralogy and alleged left Blalock anastomosis, showing right aortic arch but poor aortic filling with aortic branches hardly visible. (b) Aortogram (frontal view) showing right arch and innominate artery anastomosed to left pulmonary artery (confirmed at operation).

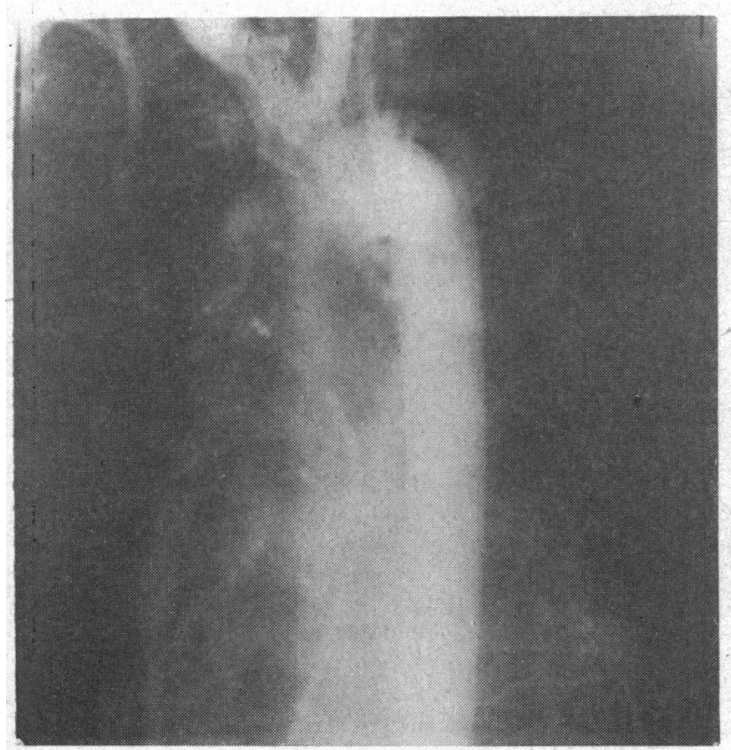

Fig. 4.-Aortogram (frontal view) showing blocked left Blalock anastomosis in patient with Fallot's tetralogy. Large collaterals arise from arch and descending aorta. A continuous murmur was audible.
They were small but more numerous, more tortuous, and arose from a wide area, particularly from the aortic arch, and from branches of the subclavian arteries. Larger collateral arteries were seen in pulmonary atresia (Fig. 7), but though the site of origin was widespread, the majority arose from the mid-thoracic part of the descending aorta in the position of the bronchial arteries. In 3 patients with pulmonary atresia two or three large vessels anatomically resembling pulmonary arteries arose directly from the descending aorta (Fig. 8 and 9).

Persistent Ductus. A large persistent ductus was the sole source of pulmonary blood flow in one patient aged 6 months with pulmonary atresia. Another, aged 9 months with Fallot's tetralogy, had a small ductus, which was not apparent on the right ventricular angiocardiogram, but was demonstrated on aortography (Fig. 2).

The Aortic Root. Aortography clearly showed the anatomy of the aortic root in all patients, whereas on the right ventricular angiocardiogram it was frequently obscured by contrast in other structures. (Fig. 10). 


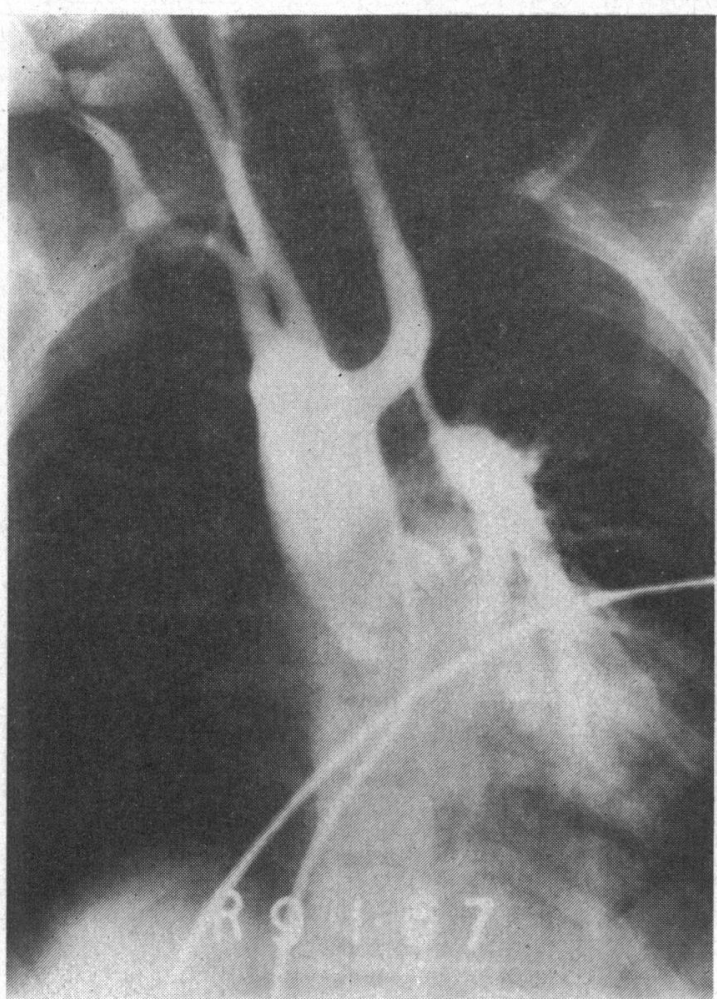

FIG. 5.-Aortogram (frontal view) in patient with left Blalock anastomosis, showing contrast medium entering left lung only.

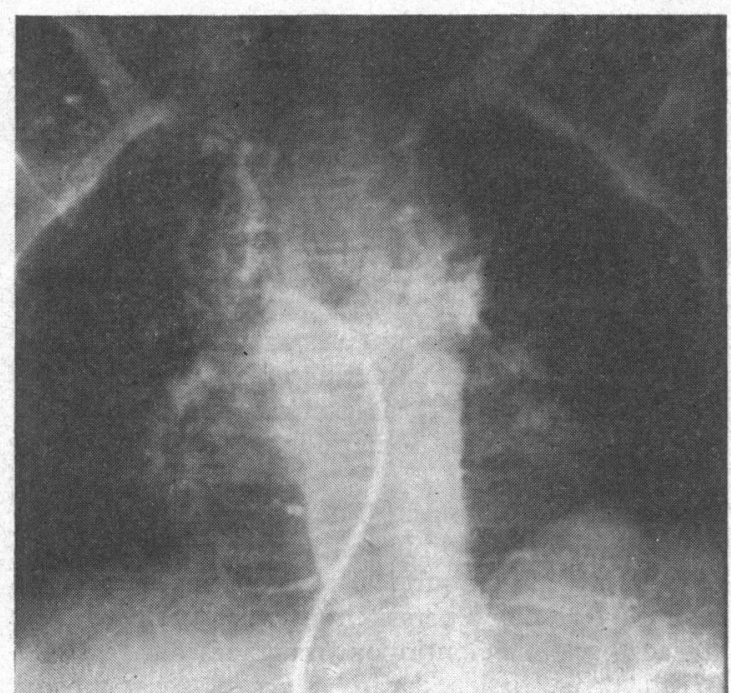

FIG. 7.-Aortogram (frontal view) in a patient with pulmonary atresia, showing numerous systemic-pulmonary collaterals with filling of a normal-sized right pulmonary artery. No continuous murmur was audible.

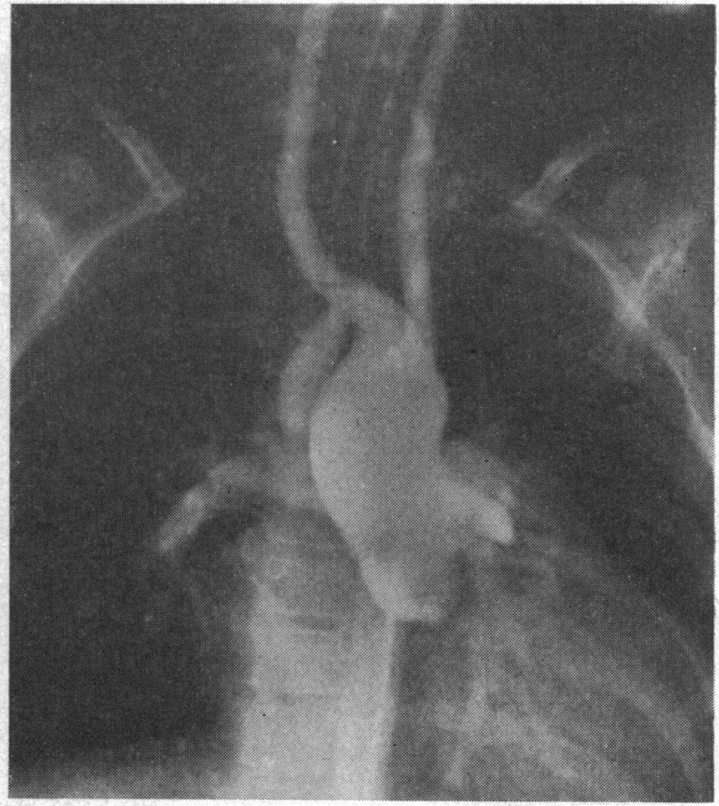

Fig. 6.-Aortogram (frontal view) in a patient with pulmonary atresia, showing both pulmonary arteries filling from a patent right Blalock anastomosis.

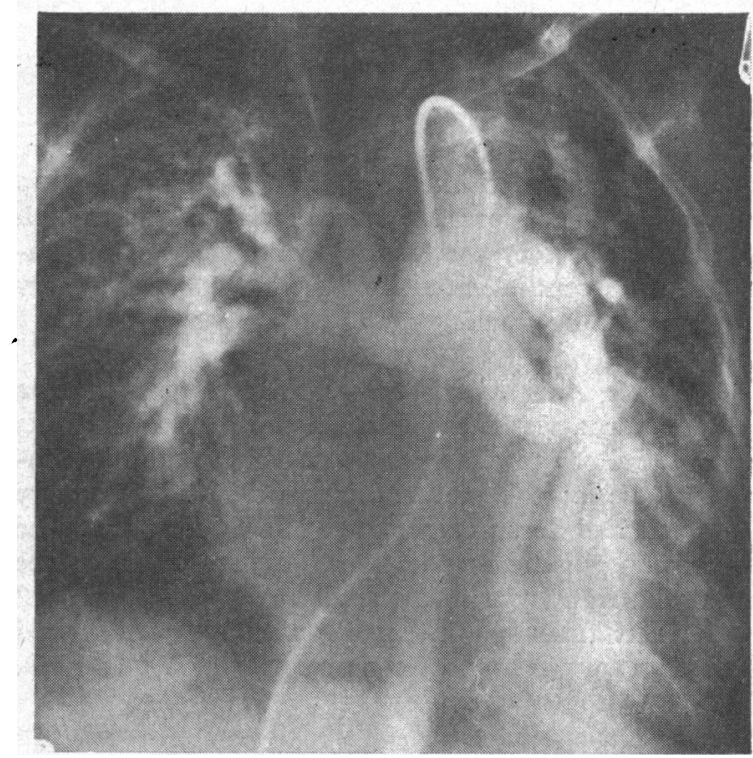

FIG. 8.-Aortogram (frontal view) in a patient with pulmonary atresia, performed by injecting into descending aorta. Large arteries arise directly from descending aorta, and enter the lungs. Their appearance suggests that they are true pulmonary arteries. 


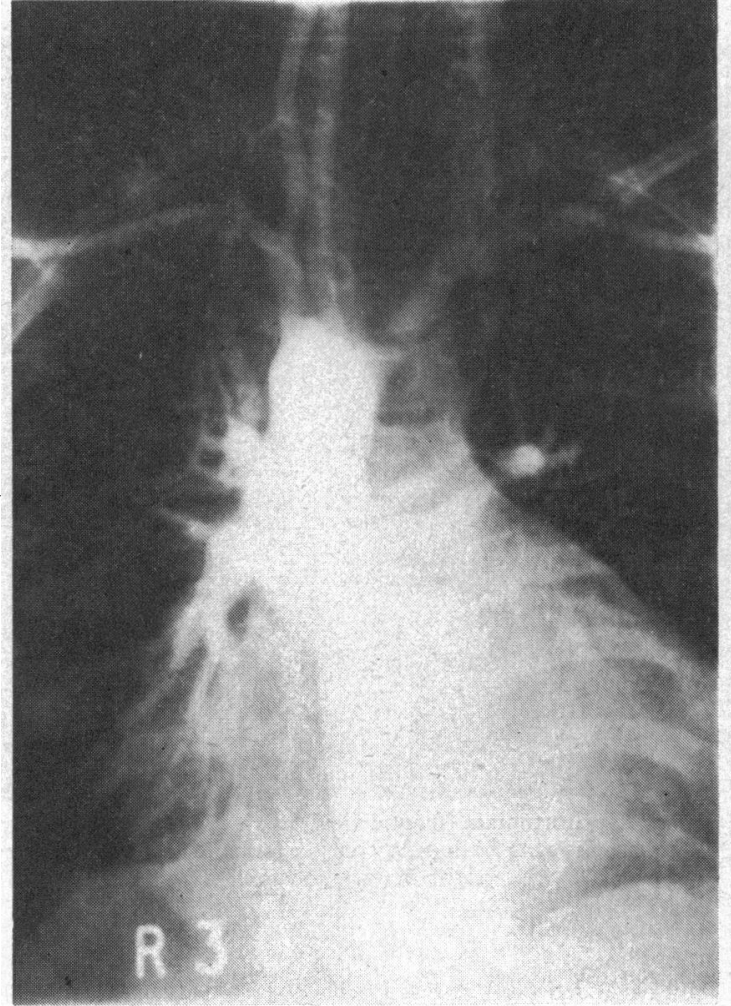

(a)

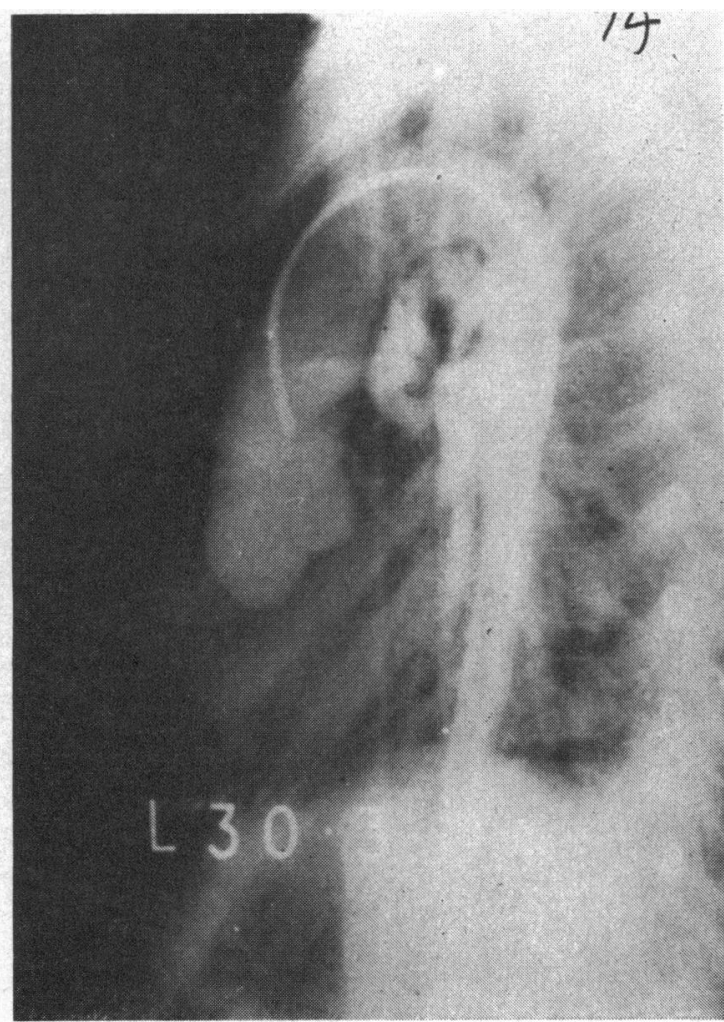

(b)

FIG. 9a and b.-Aortogram (frontal and lateral views) in patient with pulmonary atresia, showing unusually large collateral arteries perfusing pulmonary arteries.

Coronary Arteries. The coronary arteries were clearly visualized in 31 of the 40 patients. No anomaly of the coronary arteries was present, though the conal artery was usually enlarged, and the anterior descending branch of the left coronary artery tended to be small.

Aortic Arch and Brachiocephalic Arteries. The aortic arch was right-sided in 5 out of 28 patients with Fallot's tetralogy and 4 out of 10 with pulmonary atresia: the eleventh patient with pulmonary atresia had dextrocardia and a left-sided arch. The brachiocephalic arteries were often difficult to see on the right ventricular angiocardiogram, and in 2 patients abnormal findings would have been missed had an aortogram not been performed (Fig. 1 and 3).

Pulmonary Arteries in Pulmonary Atresia. In the 11 patients with pulmonary atresia, contrast medium entered the lungs from the aorta through bronchial and other collateral arteries in 7, a persistent ductus in 1, and a surgical anastomosis in 3, one of whom had extensive collaterals on the opposite side. The level at which collateral arteries joined with pulmonary arteries was variable, but one or both main pulmonary arteries were filled in 7 patients (Fig. 6 and 7). Retrograde flow into the pulmonary trunk down to the atresia was seen in three patients and in these it was possible to estimate the length of the atretic segment by superimposing the right ventricular angiocardiogram (Fig. 11). It was also noted that the pulmonary arteries were slow to opacify after the contrast injection when filling via collaterals (Somerville, 1968).

Continuous Murmur. There were 21 patients with a clinically audible continuous murmur, in all of whom a patent surgical anastomosis, large collateral arteries, or a persistent ductus were shown on the aortogram. Continuous murmurs were audible in all three patients with blocked anastomoses. One had a functioning shunt on the opposite side, but the other two had obvious large systemicpulmonary collaterals which were clearly visible on 


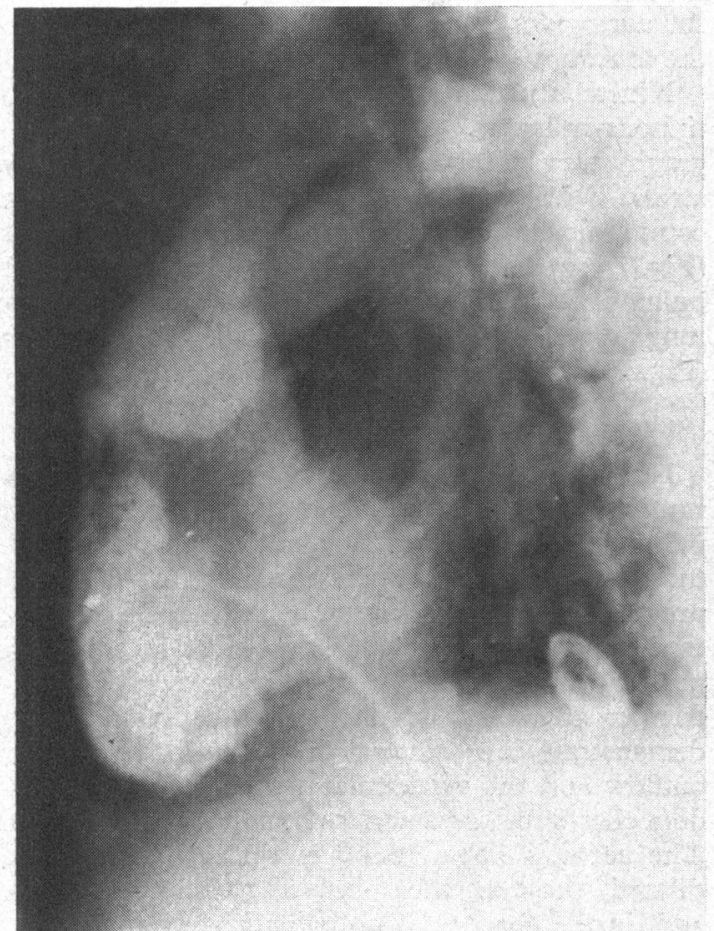

(a)

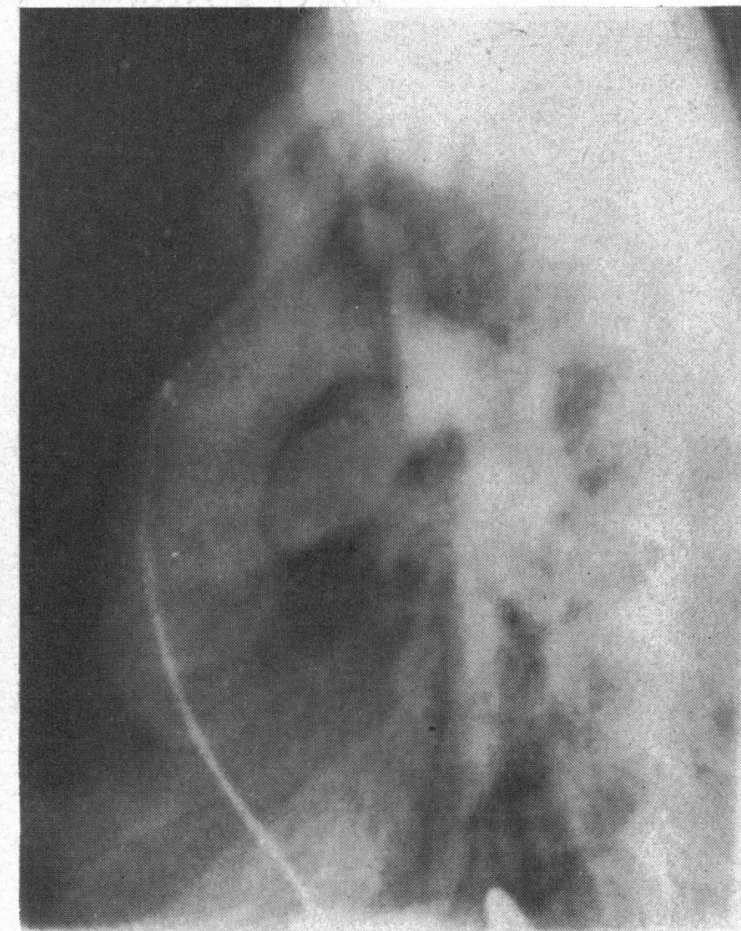

(b)

FIG. 10.-(a) Right ventricular angiocardiogram (lateral view) in a patient with Fallot's tetralogy, showing a very anterior ascending aorta. Aortic root anatomy not clear, but double-outflow right ventricle considered possible. (b) Aortogram (lateral view) showing normal posterior inclination of proximal ascending aorta and backward tilt of aortic valve plane. No evidence of double-outflow right ventricle at surgery.

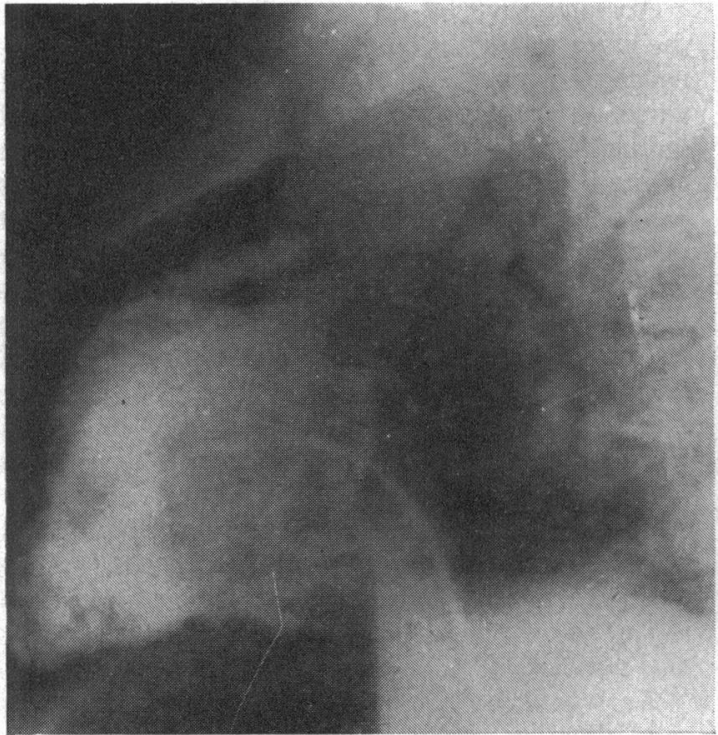

(a)

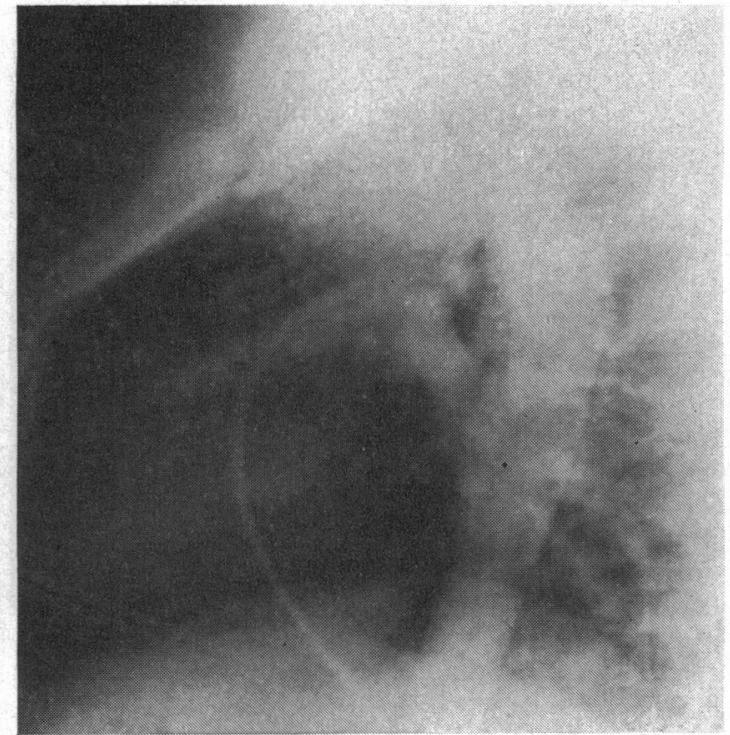

(b)

FIG. 11.-(a) Right ventricular angiocardiogram (lateral view) showing atresia at pulmonary valve level.

(b) Late frame of aortogram (lateral view) showing opacification of pulmonary arteries with retrograde filling of main pulmonary artery down to valve. Valve atresia confirmed at operation. 


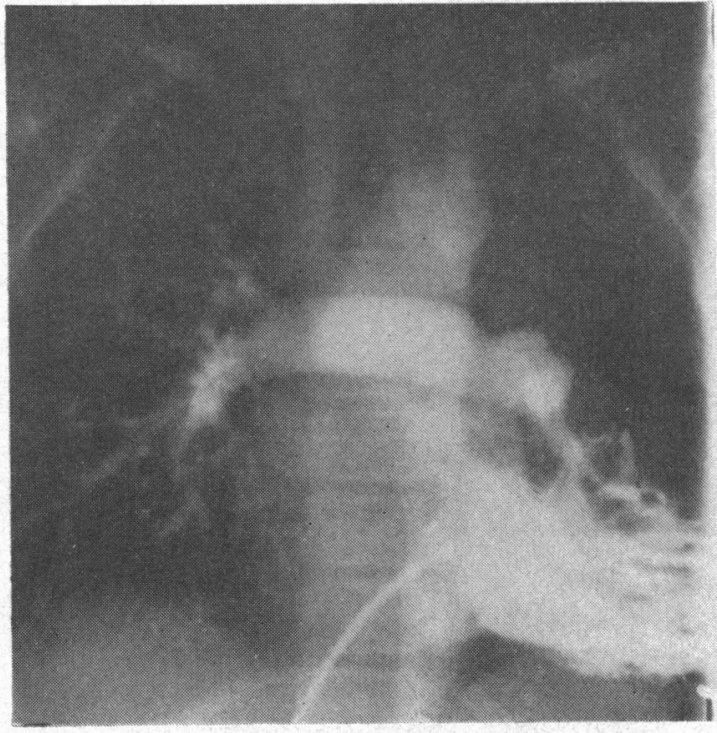

(a)

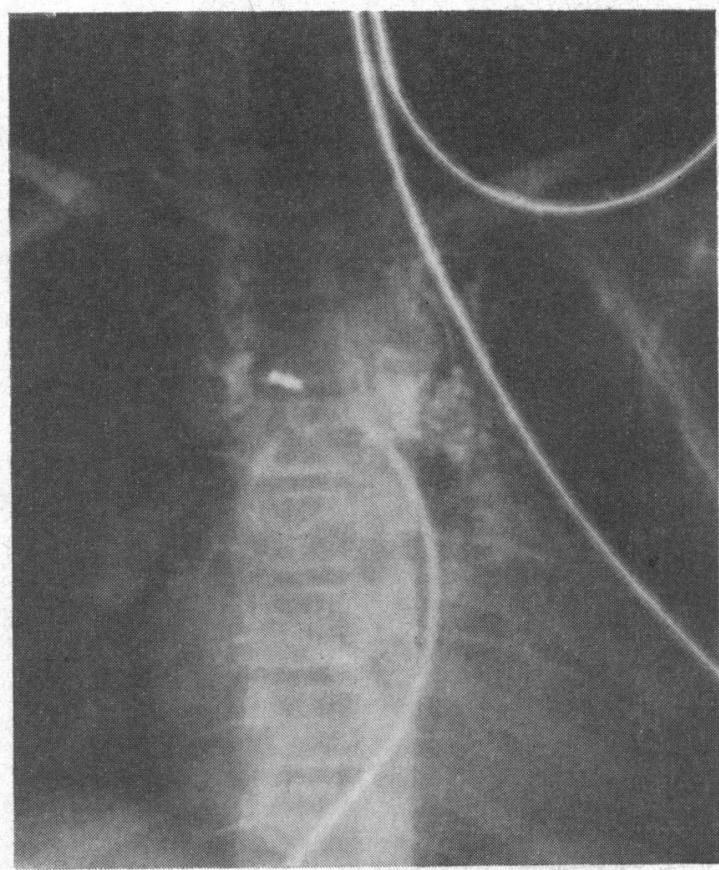

(b)

Fig. 12.-(a) Right ventricular angiocardiogram (frontal view) in patient with Fallot's tetralogy, showing infundibular stenosis, absent left pulmonary artery, and poor opacification of aorta. (b) Late frame from aortogram (frontal view) showing collateral supply to left lung. No continuous murmur was audible. the aortogram, and were presumably giving rise to the murmur.

Nineteen patients had no clinically audible continuous murmur. Unexpected findings on aortography were present in five. Two had pulmonary atresia with moderately large bronchial arteries communicating with proximal pulmonary arteries (Fig. 7), two had Fallot's tetralogy with an absent left pulmonary artery and collaterals supplying the left lung (Fig. 12), and one had a small persistent duct (Fig. 2).

\section{Discussion}

Now that total correction of Fallot's tetralogy is a routine procedure (Kirklin et al., 1959, 1965) and pulmonary atresia with favourable anatomical features is also correctable (Ross and Somerville, 1966), pre-operative demonstration of the aortic branches, especially those supplying the lung, is assuming greater importance. In these patients right ventricular angiocardiography is, of course, vital to demonstrate the anatomy of the right ventricular outflow and the ventricular septal defect, but seldom shows the aorta and its branches satisfactorily. The aorta is large, filling is variable, contrast is diluted, the ventricles obscure part of the lungs, and, in the absence of pulmonary atresia, any systemic arteries entering the lungs are hidden by the opacified pulmonary arteries. Indeed, gross anomalies of the aortic branches or a persistent ductus may be missed, aortic anatomy may not be clear, and the coronary arteries may not be visible.

Aortography is a simple additional procedure in the investigation of these patients, since the catheter usually enters the aorta easily from the right ventricle. If pulmonary atresia or a large aorto-pulmonary shunt is suspected, it is important to inject enough contrast medium, and we usually use at least $1.5 \mathrm{ml} . / \mathrm{kg}$. body weight in such patients. Anteroposterior and lateral films exposed at 6 per second in two planes are optimal, and little or no further information is gained by using a faster frame speed or cineradiography. A film series of three seconds is adequate for Fallot's tetralogy, but in pulmonary atresia, particularly in those with no duct or surgical anastomosis, a longer series of at least six seconds is needed as retrograde filling of the pulmonary trunk via collaterals may be delayed. Better demonstration of collateral arteries is also achieved by injecting contrast into the distal arch or descending aorta, if the catheter can be manipulated into this position.

As would be expected, the aortogram is most rewarding in patients with a continuous murmur (Ongley et al., 1966). Unexpected findings may also be revealed in those without, as persistent duc- 
tus and systemic-pulmonary collaterals can be clinically silent, and may be missed unless an aortogram is obtained.

In a patient with a surgical anastomosis, it is important to be certain about its patency before total correction, as a blocked anastomosis may safely be left alone. Though the continuous murmur is usually a reliable guide, it may not always be so. In two of our patients, a blocked anastomosis was accompanied by an audible continuous murmur arising from collaterals which had developed in response to the falling shunt.

Good display of the aorta and its branches may be of importance in other circumstances. Anomalies of the coronary arteries are stated to be commoner in Fallot's tetralogy than in otherwise normal hearts (Meng, Eckner, and Lev, 1965), and though no anomaly was demonstrated in the present series, it would be as well to be aware of its existence before open-heart surgery.

Double-outflow right ventricle with pulmonary stenosis is a rare lesion which can mimic Fallot's tetralogy. Since total correction of the two lesions demands a different approach, pre-operative differentiation is desirable. Important points in angiographic diagnosis of double-outflow right ventricle are anterior inclination of the ascending aorta and forward tilting of the aortic valve plane (Dayem et al., 1967). This area may be obscured by contrast in other structures on a right ventricular angiocardiogram, but is clearly shown on aortography.

In pulmonary atresia, the feasibility of radical correction depends mainly on the length of the atretic segment and the size of the pulmonary arteries. The lower end of the atresia can be shown by right ventricular angiocardiography, but an aortogram with a long film series is essential to demonstrate the pulmonary arteries and the upper end of the atretic segment.

\section{SUMMARY}

Aortography in addition to right ventricular angiocardiography has been performed in a consecu- tive series of 40 patients with Fallot's tetralogy and variants. In 36 the aorta was catheterized by the venous route. The aortogram provides information which may be vital to the surgeon but which is often unobtainable from right ventricular angiocardiography. Such information includes: (1) size, patency, and anatomy of surgical shunts; (2) site of origin, size, and number of systemic-pulmonary collaterals; (3) anatomy of aortic root in suspected double-outflow right ventricle; (4) anomalies of coronary arteries; (5) anomalies of aortic arch branches; (6) unexpected persistent ductus; and (7) anatomy of atretic segment and pulmonary arteries in pulmonary atresia.

It is suggested that aortography should be performed routinely in patients with Fallot's tetralogy and variants, who are being considered for radical corrective surgery.

\section{REFERENCES}

Dayem, M. K. A., Preger, L., Goodwin, J. F., and Steiner, R. E. (1967). Double outlet right ventricle with pulmonary stenosis. Brit. Heart f., 29, 64.

Kirklin, J. W., Ellis, F. H., McGoon, D. C., Du Shane, J. W., and Swan, H. J. C. (1959). Surgical treatment for tetralogy of Fallot by open intracardiac repair. $\mathcal{f}$. thorac. cardiovasc. Surg., 37, 22.

-, Wallace, R. B., McGoon, D. C., and DuShane, J. W. (1965). Early and late results after intracardiac repair of tetralogy of Fallot. 5-year review of 337 patients. Ann. Surg., 162, 578.

Meng, C. C. L., Eckner, F. A. O., and Lev, M. (1965). Coronary artery distribution in tetralogy of Fallot. Arch. Surg., 80, 363.

Ongley, P. A., Rahimtoola, S. H., Kincaid, O. W., and Kirklin, J. W. (1966). Continuous murmurs in tetralogy of Fallot and pulmonary atresia with ventricular septal defect. Amer. F. Cardiol., 18, 821.

Ross, D. N., and Somerville, J. (1966). Correction of pulmonary atresia with a homograft aortic valve. Lancet, 2, 1446.

Somerville, J. (1968). Angiocardiography in pulmonary atresia. Proc. Assn. European Paediatric Cardiologists, Munich. In the press. 